\title{
Akustische Erfassung von \\ Fledermäusen - Möglichkeiten und \\ Grenzen im Bau und Betrieb von \\ Windkraftanlagen
}

\section{Acoustic surveys of bats - \\ Possibilities and limitations \\ during the planning and operation \\ of wind turbines}

\section{Volker Runkel}

\section{Zusammenfassung}

Für die Planung von Eingriffen werden beinahe immer akustische Erfassungen zur Feststellung der Betroffenheit von Fledermäusen durchgeführt. Aufgrund umfangreicher, in den letzten zehn Jahren verfügbar gewordener Automatisierung der Detektoren und der Aufnahmeanalyse ist die Methode als kostengünstige und arbeitserleichternde Anwendung bei Gutachtern sehr beliebt. Die Methode hat sich in der Planungspraxis etabliert und findet sich in allen Leitfäden wieder. Durch die Möglichkeit der simultanen Erfassung mittels automatischer Detektoren, aber auch durch die Langzeiterfassung, ist die Sammlung fachlich hochwertiger Daten möglich. Dennoch bestehen nach wie vor Grenzen der akustischen Erfassung, die sich auf die erhaltenen Daten auswirken. Physikalische Einflüsse begrenzen die Erfassungsreichweite, die weiterhin art- und verhaltensspezifisch ausgeprägt ist. Für solche Einflüsse hat der Anwender keine Kontrollmöglichkeit. Der spezifische Einsatz der Detektoren hat durch Standortwahl, Einstellungen und Untersuchungsprotokoll großen Einfluss auf die erhaltenen Daten. Hier kann der Anwender den Einsatz jedoch gezielt optimieren. Dennoch unterliegen die erhaltenen Daten einer großen Streuung. Auch die Rufe der Arten sind nicht immer eindeutig bestimmbar; zwischen- und innerartliche Variabilität erschweren die Rufanalyse. Für

V. Runkel ( $\square)$

Bundesverband für Fledermauskunde Deutschland e. V., Erfurt, Deutschland

E-Mail:vrunkel@me.com 
die Bewertung stehen nur wenige Schemata zur Verfügung. Diese sind beinahe ausnahmslos subjektiv geprägt und berücksichtigen die genannten Effekte der Erfassung auf die erhaltenen Daten nicht ausreichend oder gar nicht. Daher muss der Anwender nach wie vor bei der Interpretation der Ergebnisse differenziert vorgehen. Ein gutes Verständnis der Methode ist unumgänglich, um die Daten im Hinblick auf den begutachteten Eingriff rechtssicher $\mathrm{zu}$ bewerten. Einfache pauschalisierende Aussagen sind zumeist nicht möglich. Dennoch hat die Methode große Vorteile bei der Windkraftplanung, die Grenzen sind bei korrekter Anwendung und Interpretation der Daten nur selten limitierend.

\section{Summary}

Acoustic methods are largely used for monitoring the impact of land-use changes on bats, particularly during the erection and operation of wind turbines. Over the past ten years, the automation of detectors and call analysis turned this technique into a cost-effective tool for consultants, which is now recommended by all national guidelines. Acoustic monitoring allows the collection of high quality data, e.g. when several instruments are deployed simultaneously over long periods. Nevertheless acoustic monitoring has its drawbacks and limitations. The physics of sound propagation constrains the detection range of bats, which varies with the behavior and species under consideration. These limits are beyond the control of users. Furthermore the specific use of detectors strongly influences the resulting data, for example by the choice of detector placement, recording settings and programming protocol. Here users may optimize the application of detectors to some degree. Nevertheless it is likely that the resulting data will be highly variable. Recorded echolocation calls are not always easy to identify on the species level, since inter- and intraspecific variability complicate call analysis. Only a few evaluation schemes are available, most of which are rather subjective and lack the possibility to control for the aforementioned constraints during data acquisition. Therefore, it is mandatory to follow a balanced approach when interpreting data. To make reports suitable and legally sound for environmental impact assessments, it seems mandatory to have an excellent understanding of the involved method. Simple generalized conclusions are usually impossible. Nevertheless, even when considering all limitations, acoustic monitoring techniques bear great advantages for the monitoring of bats during the planning and operation of wind turbines. When applied correctly, acoustic monitoring imposes rarely strong limitations. 


\section{$1.1 \quad$ Einleitung}

Die akustische Erfassung und Bestimmung von Fledermäusen wird im Rahmen der Windkraftplanung für unterschiedliche Fragestellungen eingesetzt. Daraus ergeben sich jeweils unterschiedliche Ansprüche an die genaue Methodik. Es gilt dabei zu unterscheiden zwischen der Voruntersuchung, die sowohl Eingriffs- und baubedingte Einflussfaktoren untersucht, sowie der späteren Höhenerfassung an der gebauten WEA, die das standortspezifische Kollisionsrisiko ermitteln soll.

Bei der Voruntersuchung werden analog zu anderen Eingriffsplanungen im Rahmen einer speziellen artenschutzrechtlichen Prüfung das Artenspektrum sowie die artspezifische Phänologie und die Betroffenheit durch Habitat- und Quartierverluste ermittelt. An Standorten mit einem komplexen Artenspektrum, zum Beispiel in Wäldern, steigen nicht nur die Anforderungen an eine akustische Untersuchung, auch stößt diese dann gegebenenfalls an ihre Grenzen. Sowohl die teilweise extrem unterschiedlichen Aktivitätsmuster einzelner Arten als auch die hohe Anzahl an ähnlich rufenden Arten erschweren die Erfassung und Bewertung. So müssen für eine zielführende Erfassung mobile und stationäre Detektoren zum Einsatz kommen, um insbesondere der Ökologie der betroffenen Arten gerecht zu werden. Weiterhin werden die im Rahmen der Voruntersuchung erhobenen Daten häufig auch für eine Abschätzung des Kollisionsrisikos für einzelne Arten beim Betrieb der geplanten WEA genutzt.

Nach dem Bau werden betriebsbedingte Einflüsse im Rahmen des akustischen Höhenmonitorings untersucht, welches zumeist in Gondelhöhe erfolgt. So soll über die festgestellte Fledermausaktivität die Häufigkeit von potenziellen Schlagopfern ermittelt werden, um daraus standortspezifische Abschaltbedingungen $\mathrm{zu}$ formulieren, in welche wichtige Umweltparameter (Temperatur, Windgeschwindigkeit) mit einfließen.

Die Einflüsse der verwendeten Technik auf die erhaltenen Daten, ebenso wie die Einflüsse der Auswertungsmethodik auf die Ergebnisse, sind dabei stark abhängig von der gewählten Fragestellung. Darüber definieren sich die Möglichkeiten, aber auch die Grenzen der akustischen Erfassung. Neben der eingesetzten Hardware, Software und Auswertungsmethodik, die der Anwender in der Regel kontrollieren kann, haben insbesondere die Schallphysik, aber auch die rufenden Fledermäuse selbst, einen großen Einfluss auf die Datenerhebung. Dies sollte bei der Bewertung von Ergebnissen immer berücksichtigt werden. Daher sollten bei jeder akustischen Fledermausuntersuchung immer gewisse Standards und Voraussetzungen erfüllt werden, damit diese sinnvoll durchgeführt werden kann (Hayes 2000; Gannon et al. 2003; Runkel 2008).

Im Folgenden wird ein Überblick der akustischen Erfassung von Fledermäusen in der Eingriffsplanung bei Windkraftprojekten gegeben. Dazu werden Einflüsse der Erfassungstechnik, der Untersuchungsmethodik, der Auswertung und der Bewertung speziell im Hinblick auf die gutachterliche Anwendung betrachtet. Es müssen die Ergebnisse, im Gegensatz zur wissenschaftlichen Grundlagenforschung, mit wirtschaftlich vertretbarem Aufwand ermittelt werden. Dabei muss sich eine ausreichende Rechtssicherheit bei der Planung und für den Betrieb ergeben. 


\subsection{Einflüsse auf die Erfassung und Bewertung von Fledermausaktivität}

\subsubsection{Physikalische Einflüsse}

Um eine Fledermaus akustisch nachzuweisen, muss sich der Ruf von der Fledermaus bis zum Detektor ausbreiten und vom Detektor auch registriert werden. Damit wirken bis zur tatsächlichen Detektion zahlreiche Faktoren auf den Ruf ein. Während der Ausbreitung der Rufe schwächen sich deren Schalldruckpegel zunehmend ab. Durch die geometrische Abschwächung halbiert sich der Schalldruckpegel $(-6 \mathrm{~dB})$ mit jeder Verdoppelung der Entfernung. Weiterhin wirkt sich die atmosphärische Abschwächung in Abhängigkeit von der Temperatur, Luftfeuchte und Frequenz dämpfend auf den Schalldruckpegel aus. Diese liegt im Bereich von $0,4-0,9 \mathrm{~dB} / \mathrm{m}$ (niedrige Frequenzen $15-30 \mathrm{kHz}$ ) und steigt auf 1,0-2,0 dB/m mit zunehmender Frequenz an (Evans et al. 1971; Bass et al. 1972; Bazley 1976). Insbesondere durch die atmosphärische Abschwächung wird die Erfassungsreichweite stark eingeschränkt. Somit wirkt sich ab einer gewissen Entfernung die Distanz deutlich stärker aus als die Ruflautstärke oder die Detektorempfindlichkeit. Wenn der Schalldruckpegel der Auslöseschwelle des verwendeten Detektors um $6 \mathrm{~dB}$ niedriger liegt und damit doppelt so empfindlich ist, gewinnt man dadurch bei höheren Abschwächungen nicht einmal $6 \mathrm{~m}$ an Reichweite (Abb. 1.1). Außerdem hat mit zunehmender Distanz die Schallkeule der Fledermaus einen größeren Einfluss auf die Erfassung.

Die Fledermaus hat durch Ruflautstärke, Frequenzverlauf des Rufs, Rufrichtung und Schallkeule starken Einfluss auf die Erfassbarkeit mit einem Detektor. Die Rufe werden bei allen Arten nicht gleich in den Raum abgestrahlt, sondern gerichtet nach vorn-unten (Jakobsen et al. 2012). Ist das Mikrofon dahingehend unpassend ausgerichtet, wird der notwendige Schalldruckpegel für eine Aufzeichnung nicht erreicht. Die Fledermaus war also für den Beobachter nicht anwesend. Die Ruflautstärken variieren von ca. $90 \mathrm{~dB}$ peSPL (re $20 \mu \mathrm{PA}$ ) (gemessen in $10 \mathrm{~cm}$ ) bis zu $136 \mathrm{~dB}$ peSPL (re $20 \mu \mathrm{PA}$ ) (gemessen in $10 \mathrm{~cm}$ vor der Fledermaus) (Waters und Jones 1995; Holderied und von Helversen 2003; Holderied et al. 2005). Innerhalb einer Art ist ein Umfang von ca. $20 \mathrm{~dB}$ zu erwarten; dies entspricht einem Faktor 10 zwischen „leise“ und „laut“. In der Regel rufen Arten, die im offenen Luftraum jagen, mit Schalldruckpegeln von 114-134 dB peSPL (re $20 \mu \mathrm{PA}$ ). Manche Arten jedoch, wie die Mopsfledermaus, schleichen sich an die Beutetiere an (Goerlitz et al. 2010; Lewanzik und Goerlitz 2018). Das bedeutet, sie nutzen leise Ortungsrufe mit teilweise unter $107 \mathrm{~dB}$ peSPL und sind damit zehn- bis 100-fach leiser als andere Arten (z. B. Gattung Nyctalus, Pipistrellus).

\subsubsection{Einfluss des Detektors}

Zur Erfassung von Fledermäusen in der Eingriffsplanung werden seit vielen Jahren akustische Detektoren eingesetzt. Lange Zeit wurden Fledermäuse im Feld durch ihre Ortungsrufe mittels manueller Mischer- oder Teilerdetektoren 
nachgewiesen (Weid und von Helversen 1987; Jüdes 1989). Bereits Mitte der 1990er Jahre wurde das Anabat-System, ein Teilerdetektor mit Nulldurchgangsanalyse, als automatischer Detektor eingesetzt (Britzke et al. 1999). Seit etwa Mitte der 2000er Jahre werden Echtzeitsysteme im Rahmen der Eingriffsplanung auch für die Dauererfassung im Freiland verwendet (Jones et al. 2000). Durch den großen Nutzen und die Arbeitserleichterung, die ein moderner Detektor im Rahmen der Eingriffs- und besonders auch der Windkraftplanung im Speziellen bieten kann, ist ein lukratives Geschäftsfeld entstanden. So stehen mittlerweile zahlreiche unterschiedliche kommerzielle Systeme zur Verfügung.

Mit Verfügbarkeit erster kommerzieller Mischer- und Teilerdetektoren wurden diese in der Wissenschaft etabliert. Man beschäftigte sich mit Eigenschaften

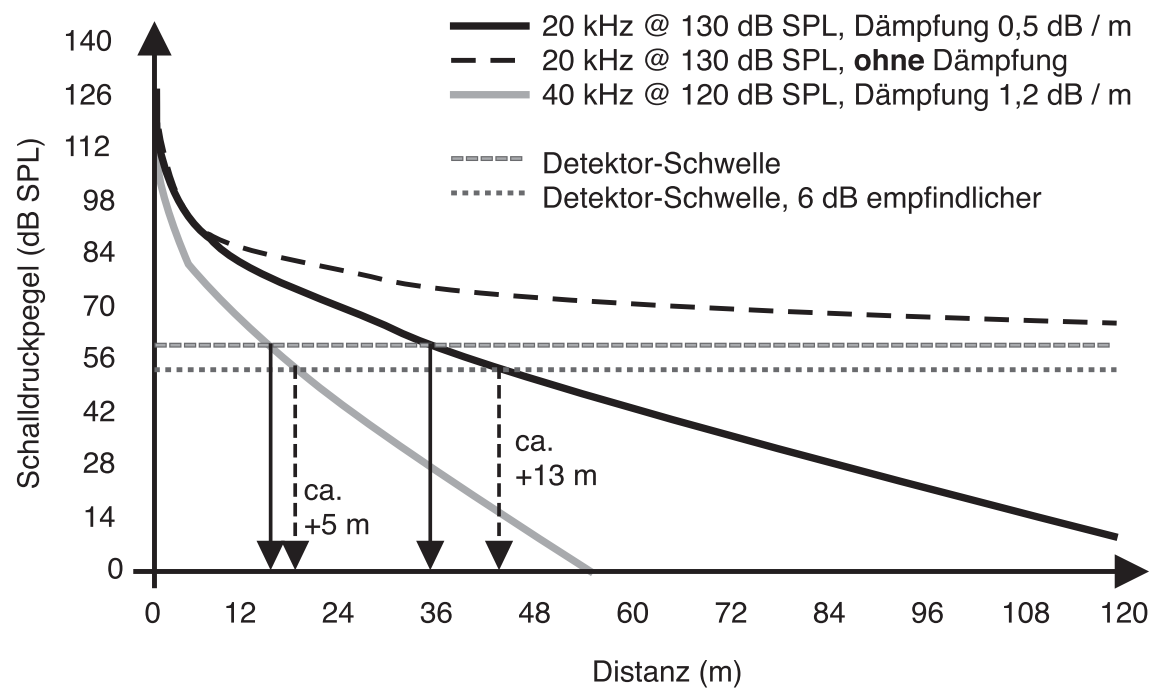

Abb. 1.1 Schematische Darstellung der Abnahme des Schalldruckpegels während der Ausbreitung in der Luft. Gezeigt ist eine schwarze Kurve für $20 \mathrm{kHz}(0,5 \mathrm{~dB} / \mathrm{m}$ Dämpfung), eine schwarze unterbrochene Kurve für $20 \mathrm{kHz}$ (ohne Dämpfung) und eine graue Kurve für $40 \mathrm{kHz}$ (1,2 db/m Dämpfung). Angepasst an Schalldruckpegel heimischer Fledermäuse liegt die Quelllautstärke der 20-kHz-Kurven bei $130 \mathrm{~dB}$ SPL (re $20 \mu \mathrm{PA}$ ), die der 40-kHz-Kurve bei $120 \mathrm{~dB}$ SPL (re $20 \mu \mathrm{PA}$ ). Dargestellt ist durch eine graue, unterbrochene Linie eine fiktive Auslöseschwelle eines Detektors. Wird diese um $6 \mathrm{~dB}$ erniedrigt (empfindlicher, grau gepunktet), hat dies aufgrund der Dämpfung dennoch keine Verdoppelung der Reichweite zur Folge

Fig. 1.1 Schematic presentation of the attenuation of the sound pressure level during the propagation of echolocation calls through the air. The black line shows data for calls at $20 \mathrm{kHz}$ with an atmospheric attenuation of $0,5 \mathrm{~dB} / \mathrm{m}$, the black-dotted line for calls at $20 \mathrm{kHz}$ without any attenuation and the gray line for calls at $40 \mathrm{kHz}$ with $1,2 \mathrm{~dB} / \mathrm{m}$ attenuation. Source levels are for European bats $130 \mathrm{~dB}$ SPL (re $20 \mu \mathrm{PA}$ ) for $20 \mathrm{kHz}$ and $120 \mathrm{~dB}$ SPL (re $20 \mu \mathrm{PA}$ ) for $40 \mathrm{kHz}$. The gray-dotted line indicates a fictive detector trigger threshold. Even when the detector sensitivity is doubled ( $+6 \mathrm{~dB}$, gray-dotted line), the detection distance is less then doubled due to atmospheric attenuation 
der Geräte und Erfassungsmodalitäten (Waters und Walsh 1994; Parsons 1996; Corben und Fellers 2000; Fenton 2000). In Studien wurden Effekte durch das verwendete Gerät, Ausrichtung des Mikrofons oder der Reichweite der Geräte untersucht (Weller und Zabel 2002; Adams et al. 2012). Auch Erfassungsmodalitäten und methodische Vergleiche wurden wiederholt im Hinblick auf mögliche Anwendungen betrachtet (O'Farrell und Gannon 1999; Johnson et al. 2002; Skalak et al. 2012).

Es zeigten sich teils starke Effekte durch die Gerätewahl. Bedingt durch technische Unterschiede ist zum Beispiel die Mikrofonempfindlichkeit unterschiedlich. Auch konnten Auswirkungen der Mikrofonausrichtung und der Installationshöhe des Detektors bei automatischer Erfassung gezeigt werden. Häufig lag bei den Vergleichen jedoch der Fokus primär auf der Erfassungsreichweite als dem - scheinbar wichtigsten - beschreibenden oder qualifizierenden Merkmal eines Detektors (Adams et al. 2012). Jedoch ist vor allem die Aufnahmequalität bei der Weiterverarbeitung der Daten entscheidend. Beim Einsatz der automatischen Rufanalyse ist die Erkennung schlecht aufgezeichneter Rufe erschwert oder nicht sinnvoll möglich.

Ein bisher wenig beachteter Aspekt ist zudem die Aufnahmesteuerung. Manche Geräte weisen variable Aufnahmeschwellen auf, die sich an die Umgebungsgeräusche anpassen. Vereinfacht gesagt wird das Gerät in einer lauten Umgebung zunehmend tauber. Aber auch ansonsten gibt es Unterschiede der Steuerung; so sind manche Triggerfunktionen nach Angaben des Herstellers zum Beispiel unempfindlicher für tieffrequente Rufe (zum Beispiel Großer Abendsegler). Die Systeme können - teils wählbare - Totzeiten nach einer Aufnahme haben und somit einen Teil der Nacht nicht aktiv sein.

Mit der zunehmenden Diversifizierung der verfügbaren Geräte in den letzten Jahren wurden jedoch nicht im selben Maße die Eigenschaften im Hinblick auf die Anwendung, insbesondere bei der Eingriffsplanung, weiter untersucht. Im RENEBAT-Projekt zum Beispiel wurden 2007 bis 2009 zu Projektbeginn die wenigen verfügbaren Geräte für den Einsatz im Gondelmonitoring untersucht (Brinkmann et al. 2011). Technische Weiterentwicklungen und neue Geräte hingegen konnten nachträglich nicht mehr mit in die Betrachtungen aufgenommen werden. Aktuelle systematische Vergleiche neu verfügbarer Lösungen existieren nicht.

\subsubsection{Einflüsse durch die Software}

Neben der verwendeten Hardware hat auch die Auswertung der erhaltenen, akustischen Daten einen entscheidenden Einfluss auf die Ergebnisse. Bei der manuellen Erfassung mittels Zeitdehnerdetektoren fallen je Begehung in der Regel nur relativ wenige einzelne Aufnahmen zur Auswertung an. Diese können in vertretbarem zeitlichen Aufwand manuell analysiert werden. Mit dem Einsatz automatischer Detektoren in Langzeiterfassungen steigen die aufgezeichneten Datenmengen jedoch massiv an. Dauererfassungen führen zu Aufnahmemengen 
von wenigen Tausend bis hin zu mehreren Hunderttausend Aufnahmen pro Jahr, Gerät und Standort. Da eine manuelle Analyse solcher Datenmengen in der Eingriffsregelung nicht adäquat bezahlt werden kann, etablierten sich in den letzten Jahren zunehmend automatische Auswertungsverfahren. Solche Systeme haben Einfluss auf die Ergebnisse und ihre Interpretation, sei es durch die Effektivität der automatischen Vermessung oder durch die Qualität der Bestimmung von Arten (Russo und Voigt 2016; Rydell et al. 2017). Die Qualität der Ergebnisse ist dabei bedingt durch verschiedene Faktoren immer noch sehr unterschiedlich. Jedoch ist sie generell ähnlich hoch wie bei menschlichen Bearbeitern (Jennings et al. 2008), ungeachtet dessen, dass ein Mensch solche Aufnahmemengen nicht ausschließlich manuell analysieren kann. Die Variabilität der Rufe einheimischer Arten ist nach wie vor nicht in Gänze bekannt oder beschrieben (Russo et al. 2018).

Insofern wirkt sich neben der Hardware auch die Qualität der automatischen Bestimmung auf die Ergebnisse aus. Jedoch bestehen in der Regel Korrekturmöglichkeiten der Anwender, die entsprechend ihrer Kenntnisse und Erfahrungen die Ergebnisse deutlich verbessern können (Fritsch und Bruckner 2014). Darüber hinaus hat die Software indirekt Auswirkungen. Benutzerfreundlichkeit, schneller Zugriff auf die Daten - auch von einzelnen Aufnahmen - und Verfügbarkeit von Auswertefunktionen wirken sich auf die Geschwindigkeit und Genauigkeit der Bearbeitung aus und sind somit im gutachterlichen Umfeld entscheidende Faktoren.

\subsubsection{Einflüsse der Untersuchungsmethode}

Akustische Untersuchungen können verschiedensten Protokollen folgen. Unterschiede finden sich in der Durchführung in Bezug auf mobile oder stationäre Erfassungen und der Häufigkeit und Dauer der Datenerhebung. Die gewählte Methode kann großen Einfluss auf die erhaltenen Daten haben. Weiterhin hat die Naturraumausstattung des untersuchten Gebiets ebenso wie die tatsächliche Fragestellung Einfluss auf die Eignung der Methoden.

Laut Fisher-Phelps et al. (2017) liefert die mobile Erfassung im Gegensatz zur stationären Erfassung bessere Ergebnisse im Hinblick auf die Aktivitätsmenge. Jedoch wurden bei der Untersuchung ein sehr spezielles Bewertungsschema und Vorgehen gewählt. Generell unterscheiden sich die Ergebnisse mobiler Transekte bereits bedingt durch die verwendete Transektmethodik (D'Acunto et al. 2018; Hogue und McGowan 2018). Andere Autoren (Stahlschmidt und Brühl 2012; Bruckner 2015; Torrez et al. 2017) kamen zu dem Ergebnis, dass Artnachweise seltener Arten und die Aktivitätsermittlung besser mittels stationärer Erfassung gelingen. Bei größeren Gebieten kann die mobile Erfassung aufgrund der besseren Gebietsabdeckung eine sinnvolle Methode sein. Mobile Erfassungen wiederum können sehr vielen verschiedenen Protokollen folgen und so zum Beispiel kontinuierlich oder als Punkt-Stopp-Transekte durchgeführt werden (Übersicht in Torrez et al. 2017; Runkel et al. 2018 mit Übersicht in Kap. 7). 
In der Praxis wird die flexible Nutzung stationärer und mobiler Erfassungsmethoden die besten Ergebnisse liefern. Artnachweise und phänologische Daten können mit gut platzierten Langzeiterfassungen gesammelt werden. Insbesondere in strukturierten Lebensräumen wie Wäldern beeinflusst der Standort eines stationären Detektors dabei die Ergebnisse (Froidevaux et al. 2014). Ereignisse in der Fläche, und somit Quartiere und Hotspots, können besser mittels mobiler Erfassung ermittelt werden.

\subsubsection{Einfluss durch die Bewertung}

Für die Beurteilung der Auswirkungen eines Eingriffs in die Landschaft müssen die erhobenen akustischen Daten in eine Bewertung überführt werden. Dazu gibt es in Deutschland in einigen Bundesländern Vorgaben zur Planung von Windkraftprojekten (Hurst et al. 2015; Dietz et al. 2016; MULE 2018). In Form von Leitfäden gelten sie in der Regel als bindend, wobei Ausnahmen möglich sind. Die Bewertungsschemata der Leitfäden beruhen dabei in aller Regel nicht auf wissenschaftlichen Ergebnissen und sind damit nicht evidenzbasiert. Vielmehr basieren sie auf subjektiver Bewertung, die von Experten vorgenommen wurde (z. B. LANU 2009; MUGV 2011). Einzig beim Gondelmonitoring ist die evidenzbasierte Anwendung ProBat in den meisten Bundesländern zumeist verpflichtend (Behr et al. 2018).

Die Basis der Bewertung der Aktivität ist bei modernen Detektoren beinahe immer die Aufnahme - vereinfacht Kontakt -, die einen oder mehrere Rufe enthalten kann. Dies ist ein sehr einfach zu ermittelndes Maß, da es keinerlei besondere Auswertung erfordert. Einzig müssen die Arten je Aufnahme ermittelt werden. Während man sich mit den technischen Aspekten der Detektoren ebenso wie mit den unterschiedlichen Untersuchungsdesigns regelmäßig beschäftigt hat, fehlt eine Diskussion der Bewertung von Aktivität beinahe gänzlich. Nur wenige Autoren haben sich mit den Möglichkeiten und Limitierungen beschäftigt (Übersicht in Frick 2013). Betrachtet man die Vielzahl an Detektoren, die allesamt unterschiedlich arbeiten, sowie die Auswirkungen des Aufbaus und der Untersuchungsmethodik, dann stellt sich die Frage, ob der Kontakt als basale Maßeinheit für Aktivität sinnvoll angewendet werden kann. Selbst in aktuell entwickelten Werkzeugen zur evidenzbasierten Bewertung wird die Kontaktzahl in Form von Aufnahmen verwendet (Lintott et al. 2017) und dabei nicht wirklich kritisch hinterfragt.

Die Daten werden für die Bewertung häufig normiert und je Stunde angegebenen. Neben den Kontakten finden sich die Aufnahmedauern als beschreibendes Maß. Seltener wird die Aktivität auf Basis von kleinen Zeitintervallen (zum Beispiel Minuten) analysiert. Akustisch erhobene Daten weisen nicht nur starke Schwankungen bedingt durch räumliche und zeitliche Variabilität der Aktivität auf, auch sind sie in der Regel nicht normalverteilt. Dies erschwert die Bewertung mittels statistischer Verfahren, und die Daten sind nicht mit einfachen statistischen Methoden zu bewerten (Lintott und Mathews 2017). 


\subsection{Möglichkeiten und Grenzen der akustischen Erfassung bei der Windkraftplanung}

Da im Rahmen der Planung von Windenergieanlagen durch die akustische Untersuchung der Eingriff rechtssicher bewertet werden muss, sollten die Möglichkeiten und die Grenzen der Methode bekannt sein. Ohne ein Verständnis und eine Diskussion der Grenzen kann der Gutachter dies eigentlich nicht leisten. Für die Untersuchung müssen die vorher genannten Faktoren mit teils starkem Einfluss auf die Ergebnisse immer berücksichtigt werden.

\subsubsection{Reichweite der Erfassung}

Einen sehr großen Einfluss auf die Ergebnisse und zugleich begrenzend für die erhaltenen Daten ist die Reichweite der Erfassung. Tiere außerhalb der Detektionsreichweite werden nicht aufgezeichnet und fehlen in der späteren Analyse der Daten. Es lässt sich dabei nicht abschätzen, wie hoch diese Anzahl ist. Neben den beschriebenen physikalischen Aspekten wirkt sich der verwendete Detektor ebenso wie die für die automatische Rufanalyse verwendete Software aus. Werden zum Beispiel leise Rufe weniger sicher erkannt, können solche Aufnahmen als „Ohne Fledermaus“ klassifiziert werden und somit bei der Bewertung fehlen.

Atmosphärische Abschwächung, Schallkeule, Frequenz und Lautstärke des Rufs haben den entscheidenden Einfluss auf die Erfassungsreichweite. In einigen Fachpublikationen werden maximale und teils mittlere Reichweiten genannt (Behr et al. 2016; Rodrigues et al. 2016). Die Autoren bleiben dabei nötige Angaben zur Witterung, aber auch zur Häufigkeit des Eintretens maximaler Reichweite schuldig. Dies bedeutet wiederum, dass diese Angaben irreführend sein können, wenn zum Beispiel die Wetterbedingungen (Temperatur, Luftfeuchte) oder die Ruflautstärken und Schallkeulen diese Werte in der Praxis nur selten erreichen lassen. Auch die Angabe einer mittleren Reichweite ist hinfällig (Behr et al. 2018), wenn nicht darstellbar ist, warum es sich um einen im Mittel zu erwartenden Wert handelt. Wird anhand der Reichweiteangaben berechnet, ob ein Erfassungsvolumen für eine Untersuchung ausreichend ist, können die Maximaangaben nur als sehr grober Richtwert genutzt werden. Bei der Diskussion sollte immer von deutlich geringeren Reichweiten ausgegangen werden. Bereits durch die täglichen Schwankungen der Parameter Luftfeuchte und Temperatur ergeben sich in der Regel Abweichungen von $\pm 10 \%$ bis maximal bis $\mathrm{zu} \pm 20 \%$ in der Detektionsreichweite (Kpersönliche Mitteilung, Jens Koblitz).

Bei der Erfassung im Vorfeld des Baus von Windenergieanlagen sollen alle vorhandenen Arten ermittelt werden, um baubedingte Auswirkungen auf diese festzustellen. Dies bedeutet, neben den eher laut rufenden Arten im offenen Luftraum müssen auch leise Arten der Gattungen Barbastella, Plecotus und Myotis erfasst werden. Diese sind jedoch häufig nur über kurze Distanzen erfassbar und 
können somit nicht immer repräsentativ aufgezeichnet werden. Eine Erhöhung der Empfindlichkeit des Detektors ist dabei nicht zwingend förderlich. Einerseits kann dies dazu führen, dass überproportional viele falsch-positive Aufnahmen (Laubheuschrecken, Umweltgeräusche) aufgezeichnet werden. Andererseits ist insbesondere in strukturierten Lebensräumen meist die Abdeckung des Mikrofons durch Vegetation erheblicher als die Empfindlichkeit des Detektors (Stahlschmidt und Brühl 2012). Sinnvoll ist es, neben bodennahen Geräten im Wald auch im Kronenbereich Detektoren einzusetzen (Plank et al. 2012).

Auch laut rufende Arten des offenen Luftraums werden nicht immer sicher aufgezeichnet. Dies liegt in dem Fall jedoch an der teils großen Flughöhe, sodass die Rufe die bodennahen Mikrofone nicht mehr mit ausreichendem Schalldruckpegel für die Auslösung einer Aufnahme erreichen oder durch Vegetation (Baumkronen) abgeschirmt werden. Insofern lässt sich zwar die Anwesenheit solcher Arten mit bodennah installierten Detektoren unter Umständen feststellen, jedoch nicht die Höhenaktivität umfassend ermitteln (Müller et al. 2013). Niedrige Aktivitäten in Bodennähe erlauben nicht den sicheren Ausschluss von Höhenaktivität, dies lässt sich nur durch ein Höhenmonitoring klären. Hohe Aktivitäten deuten auf Quartiere und immer auf ein erhöhtes Konfliktpotenzial hin. Dabei sollte mittels Referenzen immer auch klar dargelegt werden, wie eine Bewertung zur Einteilung niedrig oder hoch gelangt. Es lassen sich aus solchen Erfassungen dennoch keine detaillierten und rechtssicheren Algorithmen für den Betrieb einer zu bauenden Windindustrieanlage bereits im Vorfeld ableiten. Dazu ist immer auch die Erfassung in der relevanten Höhe nötig (Abb. 1.2).

Auch beim Gondelmonitoring ist die Reichweite ein limitierender Faktor. Bei modernen Windenergieanlagen mit Rotordurchmessern von bis zu $160 \mathrm{~m}$ liegen selbst maximale Detektionsreichweiten immer deutlich unterhalb des Radius der Rotoren. Die Verwendung der maximalen Detektionsreichweite bei der Bewertung suggeriert dann eine bessere Abdeckung, als es tatsächlich der Fall ist. Korrekt wäre es, die Detektionswahrscheinlichkeit zugrunde zu legen. Diese nimmt mit zunehmender Distanz der Fledermaus zum Detektor ab und kann neben der Detektorempfindlichkeit auch Schallkeule der Fledermaus oder andere Parameter beinhalten. Wie sich die Detektionswahrscheinlichkeit genau verhält, ist jedoch nicht untersucht. Abb. 1.3 zeigt mögliche Verläufe im Vergleich zu einem hypothetischen Rotorradius.

Ist die Erfassungsreichweite im Verhältnis zum Rotordurchmesser gering, steigt die Unsicherheit der Prognose. Da beim Gondelmonitoring jedoch insbesondere das Kollisionsrisiko betrachtet werden muss, können solche Erfassungsdefizite eine rechtssichere Bewertung erschweren oder gar unmöglich machen. Die Detektionsreichweite lässt sich dabei nicht beliebig erhöhen. Eine Erniedrigung der Auslöseschwelle des Detektors kann ebenso wie eine Erhöhung der Signalverstärkung eine starke Zunahme an Störgeräuschaufnahmen (z. B. Betriebsgeräuschen) zur Folge haben, welche ihrerseits echte Fledermausrufe maskieren und/oder die Bestimmungsgenauigkeit der automatischen Rufanalyse massiv beeinträchtigen können. Wie bereits ausgeführt, ist weiterhin der Einfluss vor allem der atmosphärischen Abschwächung bei größeren Entfernungen 
entscheidend für die Reichweite. Diese physikalische Gegebenheit lässt sich nicht kompensieren. Auch steht einer Erhöhung der Detektorempfindlichkeit momentan entgegen, dass die in Deutschland verwendete Kollisionsbewertung mittels ProBat nur mit fest vorgegebener Installation und Detektoreinstellungen arbeiten kann (Behr et al. 2018). Ein Abweichen zu höherer Empfindlichkeit für eine Reichweitenerhöhung ist daher nicht möglich.

Das Erfassungsdefizit kann jedoch durch Nutzung weiterer Mikrofone im Bereich unterhalb der Gondel bis zur unteren Rotorspitze kompensiert werden, auch wenn dann eine zusätzliche Auswertung ergänzend zur ProBat-Berechnung nötig ist. Nach wie vor können so jedoch keine Daten vom Bereich der Rotorspitzen oberhalb und seitlich der Gondel erhalten werden. Besonders leise Arten, wie die Mopsfledermaus, Arten der Gattung Myotis oder Langohrfledermäuse

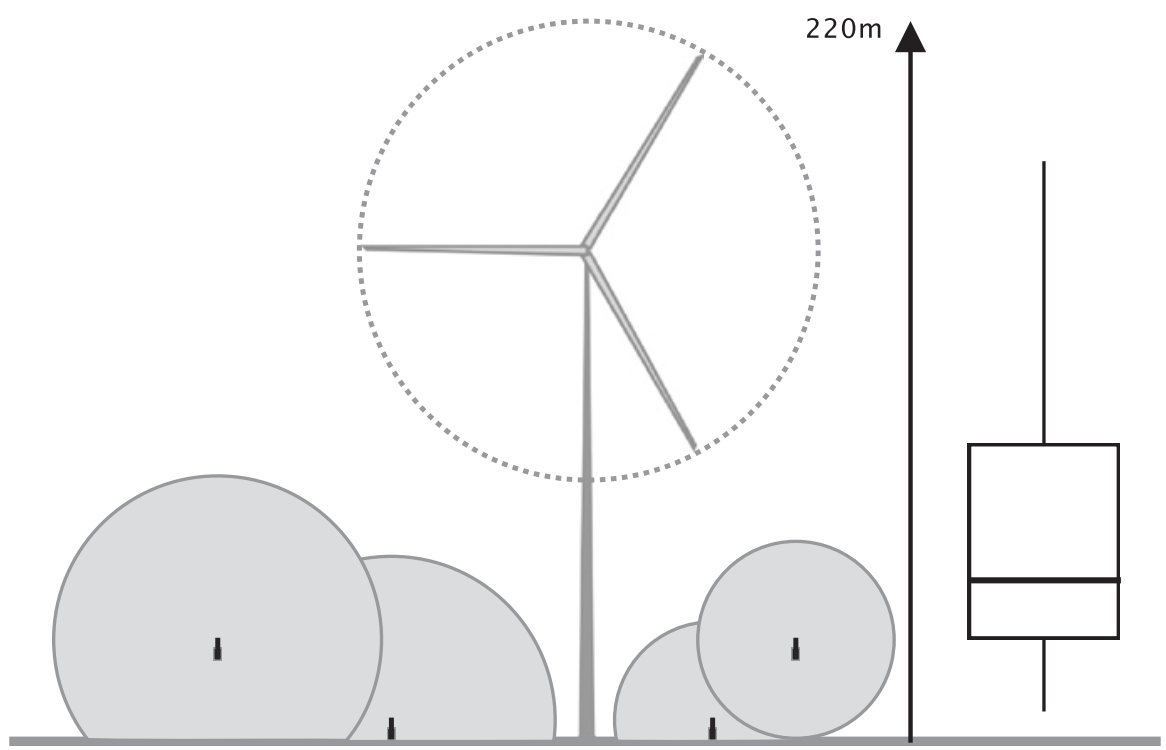

Detektion: $50 \mathrm{~m}$

Detektion: $30 \mathrm{~m}$

Abb. 1.2 Bodennahe und Höhenerfassung (Baumwipfelhöhe, ca. $30 \mathrm{~m}$ ) ist bei modernen WEA (Nabenhöhe $\geq 130 \mathrm{~m}$, Rotordurchmesser $\leq 130 \mathrm{~m}$ ) nicht ausreichend, um den potenziellen Kollisionsbereich hinreichend zu überwachen. Es wurden dazu optimistische Detektionsreichweiten von $30 \mathrm{~m}$ und $50 \mathrm{~m}$ vereinfacht als kugelförmige Erfassung geplottet. Der Boxplot (Whiskers/Box geben Quartile an) zeigt Flughöhen von Weibchen des Großen Abendseglers im Vergleich zu den Erfassungsbereichen. (Nach Roeleke et al. 2016)

Fig. 1.2 Ground- and height-based (canopy height, about $30 \mathrm{~m}$ ) acoustic recording is insufficient to cover the air space influenced by modern wind turbines (hub height $\geq 130 \mathrm{~m}$, diameter $\leq 130 \mathrm{~m}$ ). The air volume with possible collisions is not sufficiently monitored. The graph shows simplified detection ranges of $30 \mathrm{~m}$ and $50 \mathrm{~m}$, which are both conservative. The box plot (whiskers/box depict quartiles) shows flight heights of female common noctules. (After Roeleke et al. 2016) 
a

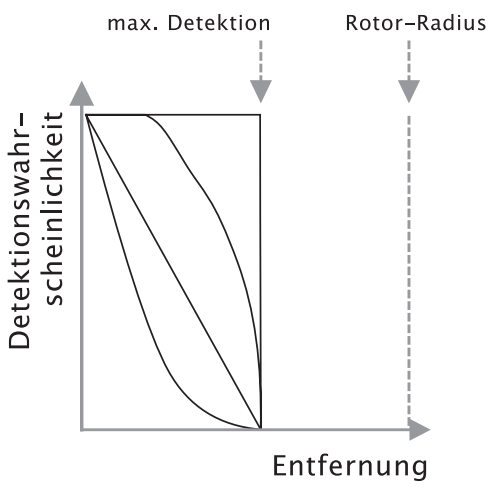

b

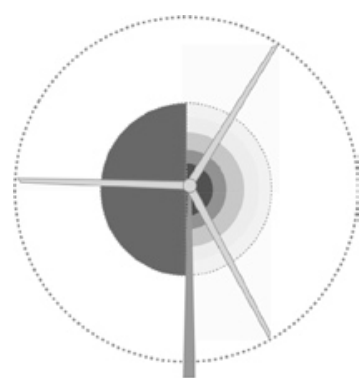

Abb. 1.3 a Detektionsreichweite in Bezug zu modernen Rotorradien. Die Detektionsreichweite sollte dabei als Wahrscheinlichkeit einer Detektion verstanden werden, die mit zunehmender Entfernung abnimmt. Gezeigt sind mehrere hypothetische Verläufe. b Gegenüberstellung von Detektionswahrscheinlichkeit gleich 1 bis maximaler Erfassungsreichweite (links) und kontinuierlicher Abnahme der Detektionswahrscheinlichkeit (rechts)

Fig. 1.3 a Detection distance is shown in relation to modern rotor diameters. The detection distance must be seen as distance dependent probability, the graph shows some hypothetical plots with various distance related decreases of probability. $\mathbf{b}$ Comparison of detection probability of one for the whole detection range (left) and one with linear decreasing probability (right)

werden in der Regel nur über wenige Meter aufgezeichnet und können somit auch bei erhöhter Empfindlichkeit mit einem zweiten Mikrofon leicht überhört werden. Insbesondere an Standorten im Wald, an denen diese Arten auftreten, besteht somit bei der akustischen Höhenerfassung immer eine Restunsicherheit, die nur durch Einsatz weiterer Mikrofone in verschiedenen Höhen am Mast oder ergänzender Methoden (Netzfang, Raumnutzungsanalyse etc.) verringert werden kann. Weiterhin scheinen Fledermäuse teilweise zur Nahrungssuche oder durch Verwechselung mit Bäumen gezielt Windkraftanlagen anzufliegen (Cryan et al. 2014; Rydell et al. 2016; Foo et al. 2017). In diesem Fall erhöht sich die Wahrscheinlichkeit eines akustischen Nachweises.

\subsubsection{Automatische und manuelle Bestimmung}

Für Gutachten im Rahmen der Eingriffsregelung werden aus Kostengründen zumeist automatische Werkzeuge zur Rufsuche und Artbestimmung eingesetzt. Diese erlauben eine objektive Ermittlung der Arten und der Aktivität. Auch wenn die verfügbaren Systeme nach wie vor teils hohe Fehlerraten aufweisen (Rydell et al. 2017; Brabant et al. 2018), sind sie als objektive Methode dennoch sinnvoll anwendbar. Insbesondere auf Gruppen- und Gattungsniveau erzielen sie teils bereits jetzt gute Ergebnisse. Wichtig ist dabei, dass bei der Rufauswertung ebenso wie beim Detektor mit einer festen Schwelle gearbeitet wird und weitere 
einstellbare Parameter des Detektors und der verwendeten Programme für die Rufsuche und Artbestimmung später im Gutachten genau dokumentiert werden. Auch bei manuellen Nachkontrollen dürfen nur die Rufe betrachtet werden, die überschwellig sind. Im Hintergrund aufgezeichnete Rufe dürfen bei der objektiven, quantitativen Auswertung nicht berücksichtigt werden, wenn sie nicht die Auslöseschwelle erreichen (Abb. 1.4). Aufnahmen eines modernen Detektors lassen Signale in dem Schalldruckpegelbereich erkennen, der sich durch den Signal-Rausch-Abstand (SNR) des Geräts ergibt. Gute Geräte weisen einen sehr guten Signal-Rausch-Abstand und damit einen großen Dynamikumfang von etwa 80 dB SPL auf. Die Auslöseschwelle liegt in der Regel deutlich über dem Grundrauschen, zumeist bei einem Eingangssignalpegel von 50-60 dB SPL (re $20 \mu \mathrm{Pa}$ ). Das bedeutet, während leisere Signale zwar noch in einer Aufnahme erkennbar sind, lösen sie alleine keine Aufnahme aus, da sie unterhalb der Auslöseschwelle liegen. Somit sind solche Signale, die über dem Rauschen liegen, aber nicht die Schwelle für eine Auslösung erreichen, zu ignorieren. Da diese Rufe nur manuell gefunden werden können, müssten ansonsten alle Aufnahmen manuell kontrolliert werden. Einzig bei der qualitativen Auswertung zum Erstellen des Artenspektrums sind solche bei Nachkontrollen gefundenen Rufe verwendbar. Werden überschwellige Rufe durch Störungen in den Aufnahmen nicht erkannt, müssen diese jedoch durch manuelle - wenigstens stichprobenartige - Kontrollen nachträglich hinzugefügt werden. Ansonsten wird ebenso die Objektivität durch eine feste Schwelle verletzt.
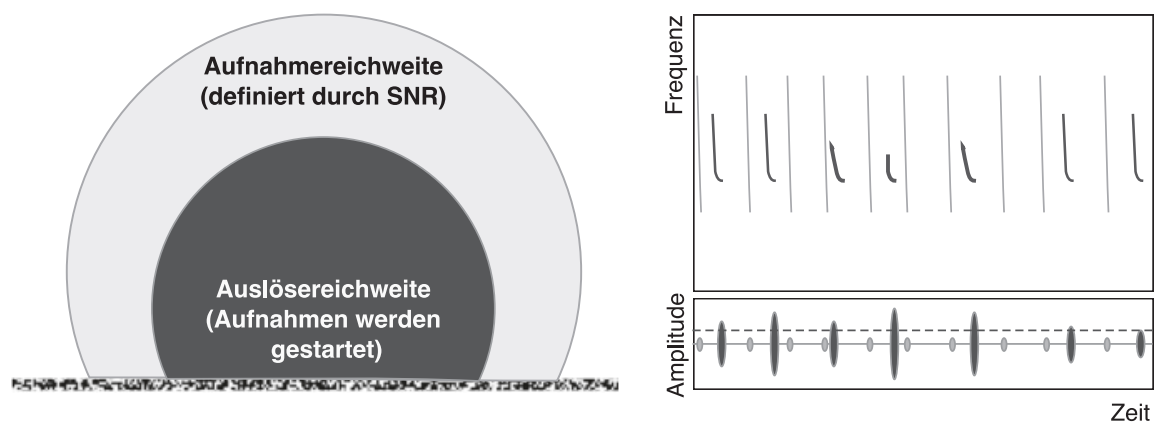

Abb. 1.4 Bei Aufnahmen moderner Detektoren mit gutem Signal-Rausch-Abstand (SNR) können Rufe aufgrund des hohen Dynamikumfangs auch deutlich unter der Auslöseschwelle noch erkannt werden. Bei der quantitativen Auswertung dürfen jedoch nur überschwellige Signale analysiert werden. Die Schwelle wird zum einen durch den Detektor und zum anderen durch die Software vorgegeben. Für eine qualitative Auswertung (Artenspektrum) können solche zu leisen, bei manuellen Kontrollen gefundenen Arten hinzugefügt werden

Fig. 1.4 The recordings of modern detectors with high signal-to-noise ratio (SNR) often contain not only calls above recording threshold but also lower amplitude calls in the background. When doing a quantitative analysis only calls above threshold should be used. The threshold is defined by detector or software and has a fixed value. In a qualitative analysis species with low intensity calls can be added manually 
Weiterhin sind einige leise rufende Arten, insbesondere der Gattung Myotis, akustisch nur schwer bestimmbar. Sowohl durch Effekte wie die Ausrichtung des Mikrofons (Ratcliffe und Jakobsen 2018) als auch durch physikalische Effekte bei der Ausbreitung (Goerlitz 2018) kann die Bestimmung weiter erschwert werden oder unmöglich sein. Insbesondere bei Voruntersuchungen in Wäldern können somit manche Arten nur bedingt untersucht werden. Die Bestimmung ist bei Arten der Gattungen Myotis und Plecotus selbst von erfahrenen Gutachtern manuell häufig nur auf Gattungsniveau möglich. So erschwert dies zusätzlich zur geringen Erfassungsreichweite den Einsatz der Methode. Bei keiner Methode kann ein Negativnachweis seltener Arten sicher erbracht werden. Dennoch liefert die akustische Erfassung gute Hinweise und kann in Kombination mit anderen Methoden wie Netzfang wertvolle Hinweise auf die Wahrscheinlichkeit des Vorkommens einer Art liefern.

Auch beim eingeschränkten Artenspektrum auf Gondelhöhe ist eine Nachkontrolle von Aufnahmen nötig. Betriebsgeräusche der Windindustrieanlage können fälschlicherweise als Fledermaus bestimmt werden oder aber die automatische Bestimmung von Rufen stören und die Fledermausrufe maskieren, sodass diese als solche nicht mehr erkannt werden (Abb. 1.5). Dies führt häufiger zu Fehlbestimmungen. Eine objektive Auswertung ist dann gegebenenfalls nicht mehr möglich und der Einsatz von Bewertungssystemen wie ProBat fraglich.

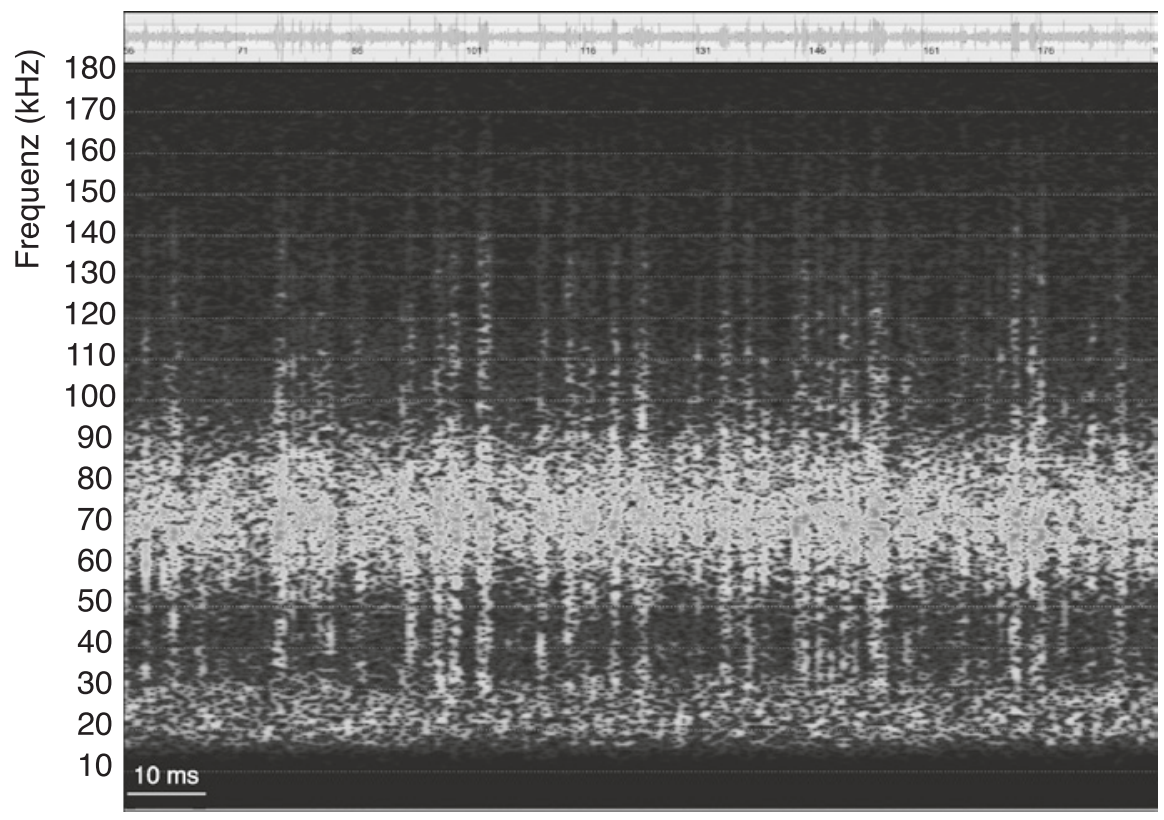

Abb. 1.5 Sonagramm eines von einer WEA erzeugten Geräuschs, das Fledermauslaute maskieren kann

Fig. 1.5 Sonagram of noise produced by a wind turbine that can potentially mask bat calls 
Auch gelingt selbst bei den scheinbar einfach zu unterscheidenden Arten des freien Luftraums die Artunterscheidung bisher automatisch nicht immer sicher. Mittels manueller Nachkontrolle ausgewählter Sequenzen lässt sich hier jedoch in der Regel nachbessern, auch wenn die Überlappung der Rufe der Gattungen Nyctalus, Vespertilio und der Art Eptesicus serotinus sehr hoch ist. Auch für die sichere Ansprache von Aufnahmen der Rauhautfledermaus, die als migrierende Art besonders windresistent ist, ist eine manuelle Nachkontrolle über den zeitlichen Zusammenhang der Aufnahmen und das Frequenzspektrum angebracht, um diese vor allem gegenüber Nachweisen der Zwergfledermaus sicher abzugrenzen.

\subsubsection{Bewertung}

Die Bewertung der Aktivität ist der entscheidende Schritt der Datenauswertung. Hierbei wird der geplante Eingriff im Hinblick auf die Verbotstatbestände des Bundesnaturschutzgesetzes $\$ 44$ eingeordnet. Für solche Betrachtungen werden bevorzugt Individuenzahlen und Populationsgröße der betroffenen Arten betrachtet, da so die Auswirkung des Eingriffs am besten rechtssicher festgestellt werden kann. Akustische Methoden können jedoch weder das eine noch das andere leisten. Individuen lassen sich anhand der Rufe nicht ermitteln. Einzig lassen sich Aufnahmen mit mehr als einem Tier bei der manuellen Analyse erkennen. Somit sind auch Abschätzungen der Populationsgröße nicht möglich.

Zur Bewertung werden zumeist die Aufnahmezahlen (auch Kontaktzahlen) verwendet. Jedoch sollte dem Anwender bewusst sein, dass diese Maßzahl sehr stark von diversen Faktoren abhängig ist. Die Fledermaus bestimmt durch ihr Verhalten bereits stark das Ergebnis. Eine kleinräumig, strukturgebunden jagende Art wird deutlich höhere Kontaktzahlen erzeugen als ein großräumiger Luftraumjäger. Auch der Standort des Detektors hat einen Einfluss auf die Anzahl der Kontakte. Steht dieser an einer Leitstruktur, erhält man höhere Aktivitäten als an einem offenen, strukturarmen Standort (Kelm et al. 2014). Ob dies eine Folge höherer Individuendichten oder nur des Verhaltens ist, lässt sich dabei nicht klar unterscheiden. Vermutlich finden Fledermäuse solche Strukturen nicht nur als Leitstruktur interessant, sondern nutzen die höhere Dichte an Beutetieren für die Jagd (Heim et al. 2015; Toffoli 2016). So kann jedoch bei bodennahen Erfassungen durch die Standortwahl das Ergebnis stark beeinflusst werden (Abb. 1.6).

Auch der verwendete Detektor hat direkt Einfluss auf die erhaltenen Aufnahmezahlen. Bedingt durch Eigenschaften wie die Empfindlichkeit, Direktionalität oder die Aufnahmesteuerung, werden beim direkten Vergleich von Geräten teils deutlich unterschiedliche Aufnahmezahlen ermittelt. Tab. 1.1 zeigt vereinfacht die Auswirkung der einzelnen Parameter. Auch gezeigt ist die Auswirkung auf die Aufnahmedauer. Diese ist ähnlich stark variabel wie die Aufnahmezahl (Kontaktzahl) (Abb. 1.6). Eine Kombination der Detektoreigenschaften mit dem Aufbau und dem Standort können jedoch gegenläufige Tendenzen ergeben. Eine nachträgliche Korrektur der Ergebnisse ist somit nicht möglich. 
a

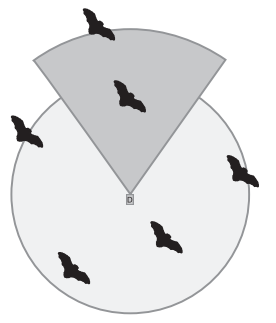

C

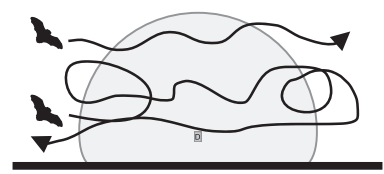

e

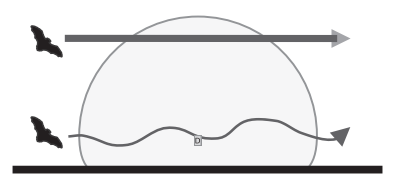

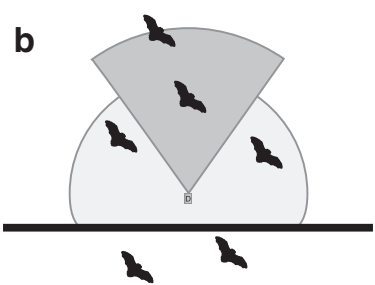

d

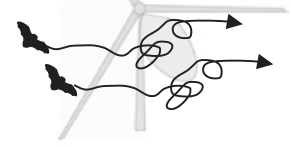

f

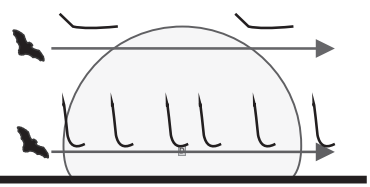

Abb. 1.6 Die Abbildungen zeigen die Auswirkung des Detektors, des Aufbaus und des Verhaltens auf die erhaltenen Anzahl Aufnahmen (=Anzahl Kontakte) (a-d). Der genaue Standort des Detektors hat durch seine räumliche Ausprägung (Schallhindernisse) starken Einfluss auf die Anzahl Aufnahmen (a, b). Unterscheiden sich die Direktionalität der Detektoren zusätzlich, kann es zu stark abweichenden Ergebnissen kommen. Geringe Aufnahmedauern erhält man beim schnellen Flug der Tiere durch den Erfassungsbereich, wohingegen ein mäandrischer Flug zu längeren Aufnahmezeiten führt (c). Beim Gondelmonitoring entscheidet die Flughöhe der Fledermaus im Hinblick auf die Detektorhöhe stark über Aufnahmezahl und Aufnahmedauer (d). Effekt auf die Aufnahmedauern, wenn Tiere, zum Beispiel bedingt durch den Abstand zur Vegetation, schneller (oben) oder langsamer (unten) fliegen und sich so unterschiedlich lange im Erfassungsraum aufhalten (e). Unterschied der Anzahl an Rufen bei Flug nahe oder entfernt von der Vegetation (f)

Fig. 1.6 The images show effects of detector, location and behaviour of bats on the resulting number of recordings (a-d). The exact location of the detector as well as the surrounding structures can in addition with different directionality of the detectors strongly influence recording count $(\mathbf{a}, \mathbf{b})$. Quick passing or meandering flight of bats both have strong influence on recording durations $(\mathbf{c})$. In nacelle monitoring the difference of flight height and detector height strongly influence counts and durations of recordings (d). Effect on recording durations due to slower (lower) or faster (upper) flight speeds of animals for example due to distance to vegetation (e). The influence of distance to clutter on the number of resulting calls is shown (f)

Die starke Streuung der Maßzahlen „Anzahl Aufnahmen“ oder „Aufnahmedauer" erschwert die Bewertung. Dies fällt besonders dann ins Gewicht, wenn der zeitliche Umfang der Datenerhebung nur wenige Nächte beträgt. Insbesondere in den Voruntersuchungen wird häufig nur an wenigen Terminen eine 
Tab. 1.1 Auswirkung der Detektoreigenschaften auf die Aufnahme-/Kontaktzahl und die Aufnahmedauern. Die Angaben sind als Tendenz zu verstehen, denn in Kombination und bedingt durch die tatsächliche Ausprägung der Eigenschaft ergeben sich sowohl kumulative als auch sich auslöschende Effekte

Tab. 1.1 Influence of detector properties on recording number and recording duration. The effects need to be seen as tendencies and can be cumulative as well as canceling in combination with each other

\begin{tabular}{|c|c|c|c|}
\hline Detektoreigenschaft & Ausprägung & $\begin{array}{l}\text { Aufnahmezahl } \\
\text { (Tendenz) }\end{array}$ & Aufnahmedauer \\
\hline \multirow{2}{*}{$\begin{array}{l}\text { Empfindlichkeit/Reich- } \\
\text { weite }\end{array}$} & Niedrig & Niedriger & Kürzer \\
\hline & Hoch & Höher & Länger \\
\hline \multirow[t]{2}{*}{ Direktionalität } & Gerichtet & Höher & Kürzer \\
\hline & Ungerichtet & Niedriger & Länger \\
\hline \multirow[t]{2}{*}{ Aufnahmesteuerung } & $\begin{array}{l}\text { Während der Rufaktivi- } \\
\text { tät }\end{array}$ & Höher & Divers \\
\hline & Feste Dauer & Niedriger & Fix \\
\hline \multirow[t]{2}{*}{ Totzeit } & Lang & Niedriger & Fix \\
\hline & Kurz & Höher & Fix \\
\hline \multirow[t]{2}{*}{ Aufnahmeschwelle } & Variabel & Niedriger & Divers \\
\hline & Fix & Höher & Fix \\
\hline
\end{tabular}

Erfassung durchgeführt. Eine längere Erfassungsdauer führt somit nicht nur zu einer besseren Bewertungsgrundlage, sondern auch dazu, dass seltene Arten oder solche, die akustisch nur schwer zu erfassen sind, zuverlässiger aufgezeichnet werden. Insofern sollten für rechtssichere Bewertungen entsprechend umfangreiche Zeiträume bereits in der Voruntersuchung erfasst werden. Auch sollte der Anwender es vermeiden, die relativ schwachen Aktivitätsindizes „Anzahl Aufnahmen“ oder „Aufnahmedauer (Sekunden)“ zu verwenden. Diese sind bei manchen Fragestellungen gegebenenfalls anwendbar. Generell erweisen sich jedoch weniger streuende und nicht so stark technikabhängige Indizes in Form von Zeitintervallen mit Aktivität als günstiger. Hier bietet sich die Zusammenfassung der Aufnahmen in 30-s- bis 10-min-Intervallen entsprechend der tatsächlichen Fragestellung an. In einer Masterarbeit wurde dazu ein Vergleich mehrerer Detektoren durchgeführt, der zu dem Ergebnis kam, dass eine Auswertung mittels eines 5-min-Zeitintervalls beinahe jegliche Streuung der Technik eliminiert (Belkin 2014).

Auch eine Beachtung der zeitlichen Verteilung der Daten kann für die Bewertung wichtig sein. So können zum Beispiel durch das Verhalten Phasen mit erhöhter Aktivität solchen Phasen mit geringer oder keiner Aktivität gegenüberstehen. Somit sind Zusammenfassungen der gesamten Aktivität nicht immer sinnvoll bei der Bewertung eines Eingriffs. 
Die bestehenden Bewertungsindizes bestehen nach wie vor zumeist auf der Anzahl Aufnahmen/Kontakten und sind auch wegen fehlender Angaben zur Technik nicht brauchbar. Sie weisen auch andere Schwachstellen auf. So wird die ermittelte Aktivität in der Regel als Mittelwert je Stunde verwendet, und es werden mehrere Klassen gebildet, die dann die Schwere des Eingriffs beurteilen. Am Beispiel eines Leitfadens des Landes Schleswig-Holstein (LANU 2009) werden die Probleme dieses Vorgehens verdeutlicht. Trotz der Veröffentlichung vor zehn Jahren findet sich das Bewertungsschema noch heute in aktuellen Gutachten auch außerhalb des Landes Schleswig-Holstein, wird also bundesweit aktiv verwendet.

Dieser Leitfaden hat in Tabelle III-9 eine Klassifizierung bestehend aus der mittleren Anzahl der aufgezeichneten Ereignisse je Untersuchungsnacht. Das „Ereignis“ ist nicht weiter definiert, wird aber in der Regel von den Gutachtern mit einer Aufnahme oder einem Kontakt gleichgesetzt. Die Bewertung des Kollisionsrisikos wird anhand sieben verschiedener Abundanzklassen festgelegt. Diese Klassen gelten gleichermaßen für alle schlaggefährdeten Arten. Damit sind Arten, die durch Flughöhe oder Jagdverhalten seltener aufgezeichnet werden, benachteiligt. Arten wie die Zwergfledermaus, die kleinräumig und eher bodennah jagt, werden hingegen bevorteilt. Ein weiteres Problem ist die Mittelwertbildung. Diese ignoriert zum einen die Phänologie, aber auch kurzzeitige Ereignisse mit hoher Aktivität. Die Mittelwertbildung ist bei akustischen Daten in der Regel nicht sinnvoll, da die Daten, insbesondere bei langen Zeiträumen, nicht normalverteilt sind. Damit ist das arithmetische Mittel nicht anwendbar.

Die Argumentationsgrundlage der Bewertung ist, dass wenige Aufnahmen automatisch auch nur eine geringe Gefährdung bedeuten. Dies findet sich so auch regelmäßig in Gutachten zu Eingriffsplanungen. Bei der Beurteilung der baubedingten Lebensraumverluste ist diese Schlussfolgerung bedingt möglich, insbesondere wenn im direkten Vergleich an anderen Standorten deutlich höhere Aktivität herrscht. Hohe Aktivität einzelner Arten (z. B. Zwergfledermaus, Pipistrellus pipistrellus) werden dann häufig noch relativiert und als intensive Jagd eines Einzelindividuums nur gering bewertet. Per se bedeuten niedrige Aktivitäten jedoch nicht zugleich eine geringe Gefährdung durch einen Eingriff. Es gibt zahlreiche, bereits genannte Faktoren, die zu einer scheinbar niedrigen Aktivität führen können. Nicht alle Arten können repräsentativ erfasst und somit auch nicht detailliert bewertet werden.

Bei Höhenmonitoring wird - in Deutschland - zumeist eine objektive Bewertung vorgenommen. In der Regel muss auf das Tool ProBat (Behr et al. 2018) zurückgegriffen werden, das als evidenzbasiertes Werkzeug verstanden werden kann. ProBat verwendet akustische Erfassungen an der Gondel, die standardisiert erhalten werden, um eine Schlagopferprognose zu erstellen. Darauf basierend werden dann je Monat und Nachtzehntel Windgeschwindigkeiten ermittelt, die als Schwellen für den Betrieb der WEA am Standort dienen. Für die Anwendung von ProBat sind strenge Voraussetzungen zu erfüllen, ansonsten können keine verlässlichen Ergebnisse ermittelt werden. Kann ProBat nicht genutzt werden, muss eine alternative Bewertung der Aktivität im Hinblick auf 
individuelle Kollisionsopfer vorgenommen werden. Dazu wird die gemessene Aktivität analog zu ProBat in Bezug zu Umweltparametern gesetzt. Im Gegensatz zu ProBat liegt dann jedoch keine statistische Korrelation von Aktivität und Schlagopfern zugrunde. Geringe Aktivität kann nicht zwingend mit geringem Kollisionsrisiko gleichgesetzt werden.

Durch Erfassungsdefizite aufgrund begrenzter Reichweite und Richtung der Fledermaus zum Detektor ist die tatsächliche Aktivität insbesondere an modernen WEA (Rotordurchmesser $\geq 120 \mathrm{~m}$ ) nicht genau bekannt, und Hochrechnungen auf die Rotorgröße sind nur bedingt möglich. Dies gilt gleichermaßen für die Anwendung von ProBat, wobei hierfür wärmeoptische Aufnahmen an einzelnen Anlagen als anekdotische Basis einer Hochrechnung dienen. Bei zunehmender Rotorgröße muss bei der Bewertung somit eine mögliche Datenunsicherheit berücksichtigt werden. So sind niedrige Aktivitäten, wenn sie sich regelmäßig wiederholen, ein klarer Hinweis für potenzielle Kollisionsopfer. Durch die möglicherweise unzureichende Erfassung können sich weiterhin Ungenauigkeiten bei der Korrelation von Aktivität mit Umweltparametern wie Windgeschwindigkeit oder Temperatur ergeben. Diese werden zumeist für Abschaltalgorithmen verwendet. Fehlt relevante Aktivität aufgrund der oben beschriebenen limitierenden Faktoren bei der akustischen Erfassung, werden somit auch falsche Abschaltungen berechnet. Dies lässt sich zum Beispiel durch wenigstens ein zusätzliches Mikrofon auf Höhe der Rotorunterkante teilweise kompensieren. Eine ausführliche Prüfung der Daten auf mögliche Erfassungslücken und der Vergleich mit im selben Naturraum erhobenen Daten sind dann weitere sinnvolle Maßnahmen für die Einschätzung möglicher Datendefizite. Bei Verdacht auf Datenlücken sollten Abschaltzeiten im Rahmen der Einschätzungsprärogative angepasst werden. Insbesondere größere zeitliche Ausnahmen von Abschaltungen sind bei fehlenden Erfassungen kritisch. Falls möglich, können durch Nachsuchen von Kollisionsopfern Verbesserungen erreicht werden, wenn der Anlagenstandort dies zulässt.

\subsection{Ansprüche an akustische Erfassung}

Die bereits eingangs erwähnten Voraussetzungen für die akustische Erfassung (Hayes 2000; Gannon et al. 2003) werden nun nochmals im Hinblick auf Gutachten in der Eingriffsplanung formuliert und ergänzt. Sowohl die Erfassung im Vorfeld der Windkraftplanung als auch beim Höhenmonitoring während des Betriebs sollte so ausgestattet sein, dass diese Aspekte alle berücksichtigt werden.

\subsubsection{Objektive Aufzeichnung}

Das verwendete Gerät muss objektiv alle Fledermäuse zuverlässig und reproduzierbar aufzeichnen. Wiederkehrende Ereignisse müssen gleichermaßen zur Aufnahme führen. Totzeiten zwischen Aufnahmen dürfen nicht auftreten. Dazu sollte die Erfassung in jeder Richtung gleich gut erfolgen und somit idealerweise 
omnidirektional sein. Die Omnidirektionalität ist insbesondere bei der bodennahen Erfassung wichtig, da dort kleinräumiges Verhalten zu erwarten ist. Beim Einsatz im offenen Luftraum sowie bei der Dauererfassung sind Kompromisse zum Beispiel im Hinblick auf die Omnidirektionalität nötig, wenn sich diese nicht vermeiden lassen (Gondeleinbau) oder wenn sie die Wetterfestigkeit erhöhen (Detektorboxen).

\subsubsection{Mikrofonempfindlichkeit}

Für die Vergleichbarkeit und Interpretation von Daten sollte das Gerät kalibriert sein und eine bekannte Grundempfindlichkeit besitzen. Die sich daraus ergebende Reichweite sollte - soweit im Hinblick auf physikalische Effekte möglich - ausreichend zur repräsentativen Untersuchung der auftretenden Arten sein. Durch simultanen Einsatz mehrerer gleich arbeitender Geräte an unterschiedlichen Messpunkten werden reichweitenbedingte Erfassungsdefizite kompensiert. Es sollte das gesamte Frequenzspektrum der zu erwartenden Fledermäuse überwacht und aufgezeichnet werden können.

\subsubsection{Ausreichende Datenqualität}

Die Bestimmung der Arten erfolgt durch Auswertung der Rufaufnahmen. Da dies auch automatisch zuverlässig erfolgen muss, sollten die Aufnahmen eine entsprechend hohe technische Qualität (Samplerate, Amplitudenauflösung, Signal-Rausch-Abstand) aufweisen. Idealerweise sind sie ohne Störungen, Echos oder andere die Bestimmung erschwerende Artefakte.

\subsubsection{Zuverlässiger Betrieb}

Die Funktionalität, insbesondere des Mikrofons, muss regelmäßig geprüft werden und das Ergebnis der Prüfung dem Anwender im Idealfall täglich vorliegen, um auf Ausfälle kurzfristig reagieren zu können. Der Detektor muss autark, das bedeutet auch ohne eine dauerhafte Stromversorgung zuverlässig aufzeichnen. Die Geräte müssen ausreichend robust sein, um typische Witterungsbedingungen im Jahresverlauf zu überstehen.

\subsubsection{Standortwahl und Erfassungsdauer}

Akustische Messungen weisen bedingt durch zeitliche und räumliche Aktivitätsmuster der Fledermäuse häufig eine sehr hohe Variabilität auf. Die Daten streuen dabei sowohl innerhalb einer Nacht als auch zwischen Nächten und im Jahresverlauf stark. Darüber hinaus haben die horizontale und vertikale Strukturierung 
des Standorts großen Einfluss auf die erhobenen Daten. Zeitliche und räumliche Faktoren müssen daher bei der Erfassung durch die Wahl geeigneter Erfassungsdauern und Standorte möglichst gut berücksichtigt werden, um eine repräsentative und aussagekräftige Erfassung zu ermöglichen.

\subsubsection{Automatisierte Artbestimmung und Datenverarbeitung}

Die automatische Rufanalyse muss so gestaltet sein, dass aufgezeichnete Rufe auch dann zuverlässig vermessen werden, wenn bis zu einem gewissen Grad Störsignale in der Aufnahme vorhanden sind. Die Ergebnisse der automatischen Artbestimmung sollten mehrere Bestimmungsebenen zulassen und eine einfache manuelle Kontrolle erlauben. Für den Austausch und die Sammlung von Daten sind einfache Schnittstellen mit ubiquitären Datenformaten sinnvoll.

\subsubsection{Neutrale Bewertung}

Die Bewertung der Ergebnisse muss neutral im Hinblick vor allem auf die verwendete Technik erfolgen. Unterschiede der verwendeten Geräte dürfen sich nicht in der Maßzahl der Aktivität niederschlagen. Die Bewertung muss art- oder artgruppenspezifisch erfolgen, um etwaige Unterschiede im Verhalten und der Ortung zu berücksichtigen. Eine pauschale Beurteilung, zum Beispiel mittels Kontaktdichten je Tag oder je Stunde, ist ebenso wie der Vergleich zwischen Arten nur bedingt oder nicht möglich. Bei der Bewertung sollte die Detektionsreichweite, wenn Bezug auf sie genommen wird, besser unter- als überschätzt werden, da sie sehr variabel ist.

\subsubsection{Dokumentation}

Sowohl die Erfassung als auch die Auswertung und die Bewertung müssen umfassend dokumentiert sein. Dazu müssen alle relevanten Informationen, so zum Beispiel die genauen Einstellungen der Geräte, durchgeführte Prüfungen (Mikrofon), aber auch alle Schritte und Parameter der Auswertung dokumentierbar sein und auch dokumentiert werden. Liegen Abweichungen von behördlichen Vorgaben vor, müssen diese ebenso überprüfbar begründet werden.

\subsubsection{Qualifizierte Bearbeiter}

Neben den technischen Aspekten der Anwendung muss der Bearbeiter eine ausreichende und umfassende Qualifizierung aufweisen. Dies ist beim Einsatz der Geräte ebenso wie bei der Auswertung und Bewertung der Daten nötig. Der hohe Automatisierungsgrad macht den qualifizierten Bearbeiter nicht überflüssig. Nur 
so können, beginnend bei der Erfassung, mögliche Probleme durch Technik, Standort oder Verhalten der Tiere erkannt und dokumentiert werden. Für die Auswertung der Daten ist eine umfassende Kenntnis der Möglichkeiten und Grenzen zwingend erforderlich.

Die Veröffentlichung wurde durch den Open-Access-Publikationsfonds für Monografien der Leibniz-Gemeinschaft gefördert.

\section{Literatur}

Adams AM, Jantzen MK, Hamilton RM, Fenton MB (2012) Do you hear what I hear? Implications of detector selection for acoustic monitoring of bats. Methods Ecol Evol 3:992998

Bass HE, Sutherland LC, Zuckerwar AJ (1972) Atmospheric absorption of sound: Analytical expressions. J Acoust Soc Am 52:2019-2021

Bazley EN (1976) Sound absorption in air at frequencies up to $100 \mathrm{kHz}$. National Physics Laboratory, Teddington

Behr O, Brinkmann R, Hochradel K, Mages J, Korner-Nievergelt F, Reinhard H, Simon R, Stiller F, Weber N, Nagy M (2018) Bestimmung des Kollisionsrisikos von Fledermäusen an Onshore-Windenergieanlagen in der Planungspraxis (RENEBAT III). $415 \mathrm{~S}$

Behr O, Brinkmann R, Korner-Nievergelt F, Nagy M, Niermann I, Reich, M, Simon R (2016) Ergebnisbericht des Forschungsvorhabens „Reduktion des Kollisionsrisikos von Fledermäusen an Onshore-Windenergieanlagen (RENEBAT II)“. 374 S

Belkin B (2014) Vergleich verschiedener Horchkisten zur akustischen Erfassung von Fledermauskontakten bei der Planung von Windenergieanlagen. Master thesis. Universität Oldenburg. $84 \mathrm{~S}$

Brabant R, Laurent Dolap U, Degraer S, Poerink BJ (2018) Comparing the results of four widely used automated bat identification software programs to identify nine bat species in coastal Western Europe. Belg J Zool 148:1-11

Brinkmann R, Behr O, Niermann I, Reich M (2011) Entwicklung von Methoden zur Untersuchung und Reduktion des Kollisionsrisikos von Fledermäusen an Onshore-Windenergieanlagen. Umwelt und Raum. Bd 4. Cuvillier Verlag, Göttingen. $470 \mathrm{~S}$

Britzke ER, Murray KL, Hadely BM, Robbins LW (1999) Measuring bat activity with the Anabat II system. Bat Res News 40:1-3

Bruckner A (2015) Recording at water bodies increases the efficiency of a survey of temperate bats with stationary, automated detectors. Mammalia 80:196-199

Corben C, Fellers GM (2000) Choosing the correct bat detector a reply. Acta Chiropterol 2:253256

Cryan PM, Gorresen PM, Hein CD, Schirmacher MR, Diehl RH, Huso MM, Hayman DTS, Fricker PD, Bonaccorso FH, Johnson DH, Heist K, Dalton DC (2014) Behavior of bats at wind turbines. Proc Nat Acad of Sci USA 111:15126-15131

D'Acunto LE, Pauli BP, Moy M, Johnson K, Abu-Omar J, Zollner PA (2018) Timing and technique impact the effectiveness of road-based, mobile acoustic surveys of bats. Ecol Evol 8:3152-3160

de Torrez ECB, Wallrichs MA, Ober JK, McCleery RA (2017) Mobile acoustic transects miss rare bat species: implications of survey method and spatio-temporal sampling for monitoring bats. PeerJ 5:e3940

Dietz M, Krannich E, Weitzel M (2016) Arbeitshilfe zur Berücksichtigung des Fledermausschutzes bei der Genehmigung von Windenergieanlagen (WEA) in Thüringen. $121 \mathrm{~S}$

Evans LB, Bass HE, Sutherland LC (1971) Atmospheric absorption of sound: theoretical predictions. J Acoust Soc Am 51:1565-1575 
Fenton MB (2000) Choosing the correct bat detector. Acta Chiropterol 2:215-224

Fisher-Phelps M, Schwilk D, Kingston T (2017) Mobile acoustic transects detect more bat activity than stationary acoustic point counts in a semi-arid and agricultural landscape. J Arid Environm 136:38-44

Foo CF, Benett VJ, Hale AM, Korstian JM, Schildt AJ, Williams DA (2017) Increasing evidence that bats actively forage at wind turbines. PeerJ 5:e3985

Frick WF (2013) Acoustic monitoring of bats, considerations of options for long-term monitoring. Therya 4:69-78

Fritsch G, Bruckner A (2014) Operator bias in software-aided bat call identification. Ecol Evol 4:2703-2713

Froidevaux JSP, Zellweger F, Bollmann K, Obrist MK (2014) Optimizing passive acoustic sampling of bats in forests. Ecol Evol 4:4690-4700

Gannon WL, Sherwin RE, Haymond S (2003) On the importance of articulating assumptions when conducting acoustic studies of habitat use by bats. Wildl Soc Bull 31:45-61

Goerlitz HR, ter Hofstede HM, Zeale MR, Jones G, Holderied MW (2010) An aerial-hawking bat uses stealth echolocation to counter moth hearing. Current Biol 20:1568-1572

Goerlitz HR (2018) Weather conditions determine attenuation and speed of sound: Environmental limitations for monitoring and analyzing bat echolocation. Ecol Evol 8:5090-5100

Hayes JP (2000) Assumptions and practical considerations in the design and interpretation of echolocation-monitoring studies. Acta Chiropterol 2:225-236

Heim O, Treitler JT, Tschapka M, Knörnschild M, Jung K (2015) The importance of landscape elements for bat activity and species richness in agricultural areas. PLoS ONE 10:1-14

Hogue AS, McGowan AT (2018) Comparison of driving transect methods for acoustic monitoring of bats. In Bats. InTech, 1-15

Holderied MW, Korine C, Fenton B, Parsons S, Robson S, Jones G (2005) Echolocation call intensity in the aerial hawking bat Eptesicus bottae (Vespertilionidae) studied using stereo videogrammetry. J Exp Biol 208:1321-1327

Holderied MW, von Helversen O (2003) Echolocation range and wingbeat period match in aerial-hawking bats. Proc R Soc London 270:2293-2299

Hurst J, Balzer S, Biedermann M, Dietz M, Höhne E, Karst I, Petermann R, Schorcht W, Steck C, Brinkmann R (2015) Erfassungsstandards für Fledermäuse bei Windkraftprojekten in Wäldern. Nat Landsch 90:157-169

Jakobsen L, Ratcliffe JM, Surlykke A (2012) Convergent acoustic field of view in echolocating bats. Nature 493:93-96

Jennings N, Parsons S, Pocock MJO (2008) Human vs. machine: identification of bat species from their echolocation calls by humans and by artificial neural networks. Can J Zool 86:371-377

Johnson JB, Menzel MA, Edwards JW, Ford WM (2002) A comparison of 2 acoustical bat survey techniques. Wildl Soc Bull 30:931-936

Jones G, Vaughan N, Parsons S (2000) Acoustic identification of bats from directly sampled and time expanded recordings of vocalizations. Acta Chiropterol 2:155-170

Jüdes U (1989) Erfassung von Fledermäusen im Freiland mittels Ultraschalldetektor. Myotis 27:27-38

Kelm DH, Lenski J, Kelm V, Toelch U, Dziock F (2014) Seasonal bat activity in relation to distance to hedgerows in an agricultural landscape in Central Europe and implications for wind energy development. Acta Chiropterol 16:65-73

LANU (2009) Empfehlungen zur Berücksichtigung tierökologischer Belange bei Windenergieplanungen in Schleswig-Holstein. Schriftenreihe LANU SH 13:1-93

Lewanzik D, Goerlitz HR (2018) Continued source level reduction during attack in the low-amplitude bat Barbastella barbastellus prevents moth evasive flight. Funct Ecol 32:1251-1261 
Lintott PR, Davison S, Van Breda J, Kubasiewicz L, Dowse D, Daisley J, Mathews F (2017) Ecobat: an online resource to facilitate transparent, evidence-based interpretation of bat activity data. Ecol Evol 8:935-941

Lintott PR, Mathews F (2017) Basic mathematical errors may make ecological assessments unreliable. Biodiv Conserv 27:1-3

MUGV (2011) Erlass des Ministeriums für Umwelt, Gesundheit und Verbraucherschutz. Beachtung naturschutzfachlicher Belange bei der Ausweisung von Windeignungsgebieten und bei der Genehmigung von Windenergieanlagen. $6 \mathrm{~S}$

MULE (Ministerium für Umwelt, Landwirtschaft und Energie) (2018) Leitfaden Artenschutz an Windenergieanlagen in Sachsen-Anhalt. $47 \mathrm{~S}$

Müller J, Brandl R, Buchner J, Pretzsch H, Seifert S, Straetz C, Fenton B (2013) From ground to above canopyBat activity in mature forests is driven by vegetation density and height. For Ecol Manag 306:179-184

O'Farrell MJ, Gannon WL (1999) A comparison of acoustic versus capture techniques for the inventory of bats. J Mamm 80:24-30

Parsons S (1996) A comparison of the performance of a brand of broad-band and several brands of narrow-band bat detectors in two different habitat types. Bioacoustics 7:33-43

Plank M, Fiedler K, Reiter G (2012) Use of forest strata by bats in temperate forests. J Zool 286:154-162

Ratcliffe JM, Jakobsen L (2018) Dont believe the mike: behavioural, directional, and environmental impacts on recorded bat echolocation call measures. Can J Zool 96:283-288

Rodrigues L, Bach L, Dubourg-Savage MJ, Goodwin J, Harbusch C (2016) Leitfaden für die Berücksichtigung von Fledermäusen bei Windenergieprojekten: Überarbeitung 2014. Eurobats Publication Series 6.73 S

Roeleke M, Blohm T, Kramer-Schadt S, Yovel Y, Voigt CC (2016) Habitat use of bats in relation to wind turbines revealed by GPS tracking. Sci Rep 6:28961

Runkel V (2008) Mikrohabitatnutzung syntoper Waldfledermäuse. PhD thesis. Universität Erlangen-Nürnberg

Runkel V, Gerding G, Marckmann U (2018) Handbuch: Praxis der akustischen Fledermauserfassung. Hamburg, tredition

Russo D, Ancillotto L, Jones G (2018) Bats are still not birds in the digital era: echolocation call variation and why it matters for bat species identification. Can J Zool 96:63-78

Russo D, Voigt CC (2016) The use of automated identification of bat echolocation calls in acoustic monitoring: a cautionary note for a sound analysis. Ecol Ind 66:598-602

Rydell J, Bogdanowicz W, Boonman A, Pettersons S, Suchecka E, Pomorski JJ (2016) Bats may eat diurnal flies that rest on wind turbines. Mamm Biol 81:331-339

Rydell J, Nyman S, Eklöf J, Jones G, Russo D (2017) Testing the performances of automated identification of bat echolocation calls: a request for prudence. Ecol Ind 78:416-420

Skalak SL, Sherwin RE, Brigham RM (2012) Sampling period, size and duration influence measures of bat species richness from acoustic surveys. Methods Ecol Evol 3:490-502

Stahlschmidt P, Brühl CA (2012) Bats as bioindicators - the need of a standardized method for acoustic bat activity surveys. Methods Ecol Evol 3:503-508

Toffoli R (2016) The importance of linear landscape elements for bats in a farmland area: the influence of height on activity. J Landsc Ecol 9:49-62

Waters DA, Jones G (1995) Echolocation call structure and intensity in five species of insectivorous bats. J Exp Biol 198:475-489

Waters DA, Walsh AL (1994) The influence of bat detector brand on the quantitative estimation of bat activity. Bioacoustics 5:205-221

Weid R, von Helversen O (1987) Ortungsrufe europäischer Fledermäuse beim Jagdflug im Freiland. Myotis 25:5-27

Weller TJ, Zabel CJ (2002) Variation in bat detections due to detector orientation in a forest. Wildl Soc Bull 30:922-930 
Open Access Dieses Kapitel wird unter der Creative Commons Namensnennung 4.0 International Lizenz (http://creativecommons.org/licenses/by/4.0/deed.de) veröffentlicht, welche die Nutzung, Vervielfältigung, Bearbeitung, Verbreitung und Wiedergabe in jeglichem Medium und Format erlaubt, sofern Sie den/die ursprünglichen Autor(en) und die Quelle ordnungsgemäß nennen, einen Link zur Creative Commons Lizenz beifügen und angeben, ob Änderungen vorgenommen wurden.

Die in diesem Kapitel enthaltenen Bilder und sonstiges Drittmaterial unterliegen ebenfalls der genannten Creative Commons Lizenz, sofern sich aus der Abbildungslegende nichts anderes ergibt. Sofern das betreffende Material nicht unter der genannten Creative Commons Lizenz steht und die betreffende Handlung nicht nach gesetzlichen Vorschriften erlaubt ist, ist für die oben aufgeführten Weiterverwendungen des Materials die Einwilligung des jeweiligen Rechteinhabers einzuholen. 